\title{
Effect of nitrogen and iron in carbon nanowalls on oxygen reduction reaction
}

\author{
Moeka Taniguchi ${ }^{a}$, Ryo Yoshie ${ }^{a}$, Kazuma Akikubo ${ }^{b}$, Akira Tateno ${ }^{b}$, Kozue Hotozuka ${ }^{b}$, Norihito Kawaguchi ${ }^{b}$, \\ Takashi Uchida $^{c}$, Makoto Tanimura ${ }^{\mathrm{d}}$, Masaru Tachibana ${ }^{\mathrm{a}, *}$ \\ a Department of Materials System Science, Yokohama City University, 22-2 Seto, Kanazawa-Ku, Yokohama, 236-0027, Japan \\ b Corporate Research and Development, IHI Corporation, 1, Shin-nakahara-cho, Isogo-ku, Yokohama, 235-8501, Japan \\ ' Bio-Nano Electronics Research Centre, Toyo University, 2100 Kujirai, Kawagoe-Shi, Saitama-Ken, 350-0815, Japan \\ d Instrumental Analysis Center, Yokohama National University, 79-5 Tokiwadai, Hodogaya-Ku, Yokohama, 240-8501, Japan
}

\section{A R T I C L E I N F O}

Article history:

Received 30 November 2018

Received in revised form 11 February 2019

Accepted 13 March 2019

Available online $\mathrm{xxx}$

Keywords:

Carbon nanowalls

Fe-N-doped carbon catalyst

Oxygen reduction reaction

Trace iron

Fuel cell

\begin{abstract}
A B S T R A C T
The effect of nitrogen $(\mathrm{N})$ and iron $(\mathrm{Fe})$ on oxygen reduction reaction (ORR) was investigated by using carbon nanowalls (CNWs) as carbon matrix. The dopings of $\mathrm{N}$ and Fe into CNWs are carried out by $\mathrm{N}$ plasma and $\mathrm{Fe}$ sputtering, respectively. The onset potentials corresponding to ORR activities are evaluated from steady-state curve of cyclic voltammogram. The composition and structure of CNW catalysts before and after ORR measurements are characterized by X-ray photoelectron spectroscopy (XPS). The onset potential increases in the order of non-, N- and Fe-N-doped CNW catalysts. The ORR activity for N-doped CNW catalysts is ascribed to pyridinic $\mathrm{N}$ stabilized near the surface of carbon matrix. The higher ORR activity for Fe-N-doped CNW catalysts is attributed to trace Fe-N rather than pyridinic $\mathrm{N}$ near the surface. Additionally, it is shown that such ORR activities due to pyridinic $\mathrm{N}$ and $\mathrm{Fe}-\mathrm{N}$ near the surface are further promoted by $\mathrm{Fe}$ and $\mathrm{Fe}-\mathrm{N}$ incorporated beneath the surface. These knowledges provide effective strategy for the synthesis of Fe-N-doped carbon catalysts with higher ORR activities.
\end{abstract}

(C) 2019 .

\section{Introduction}

Proton exchange membrane fuel cells (PEMFCs) have attracted much attention as green renewable energy devices in view of the increasing global energy demand and environmental impact of traditional energy sources. In such fuel cells, oxygen reduction reaction (ORR) at cathode is a key electrochemical reaction, due to its rate-limiting reaction associated with the complex reaction pathways and sluggish electron-transfer kinetics. The most effective electrode catalyst for the ORR is made of highly dispersed platinum (Pt) or Pt alloys supported on carbon black. However, the Pt has crucial disadvantages such as the low natural abundance, high costs, and lack of long-term stability, which have hindered wide applications of fuel cells. Therefore, the development of non-Pt or inexpensive catalysts for ORR is strongly desired for practical applications of PEMFCs [1,2].

Carbon-based materials have been found to exhibit apparent ORR activity and serve as a promising candidate to replace Pt for ORR catalysts. These carbon catalysts are generally doped with heteroatoms such as $\mathrm{N}$ and $\mathrm{S}, \mathrm{B}, \mathrm{P}$, and the activity is further enhanced by the incorporation of transition metals such as $\mathrm{Fe}$ and Ni, Co [3,4]. Among these, $\mathrm{Fe}-\mathrm{N}$-doped carbon catalysts stand out because of their remarkable ORR activity that even rivals that of commercial $\mathrm{Pt} / \mathrm{C}$ catalysts. The knowledge on active sites is required for the rational de-

\footnotetext{
* Corresponding author.

Email address: tachiban@yokohama-cu.ac.jp (M. Tachibana)
}

sign and preparation of catalysts with higher ORR activity for the practical applications. However, there are still debates on what are the real active sites and whether Fe only promotes the formation of active sites or directly participates in catalyzing ORR [5-8].

One reason why the consensus on active sites is not reached among researchers is the inhomogeneity and complexity of structure and composition in the catalysts which are generally synthesized by the pyrolysis of selected precursors. These leads to the drastic variation of the ORR activity, since it strongly depends not only the concentrations and molecular configurations of active sites (dopants) but also porosity, surface accessibility, and electrical conductivity of the carbon matrix. Thus, well-defined carbon materials need to be used as carbon matrix for the identification of active sites. The other reason is the lack of the characterization for real or stable states of catalysts during ORR. The catalysts are often modified due to the elution of the surface elemental species in acid medium during ORR. Thus, to identify the real active sites in catalysts during ORR, the stable or steady states of catalysts during or after ORR have to be characterized by X-ray photoelectron spectroscopy (XPS) etc.

In this paper, we show the effect of $\mathrm{N}$ and Fe on ORR by using well-defined carbon nanowalls (CNWs) as carbon matrix, which are synthesized by using plasma-enhanced chemical vapor deposition (PECVD)/sputtering hybrid system reported previously [9]. CNWs are vertical aligned carbon sheets which consist of nano-graphite domains with uniform and high quality. The structural feature means that CNWs have a lot of domain boundaries which are effective as adsorption or doping sites. Thus, CNWs are suitable as carbon matrix for the understanding of active sites. 


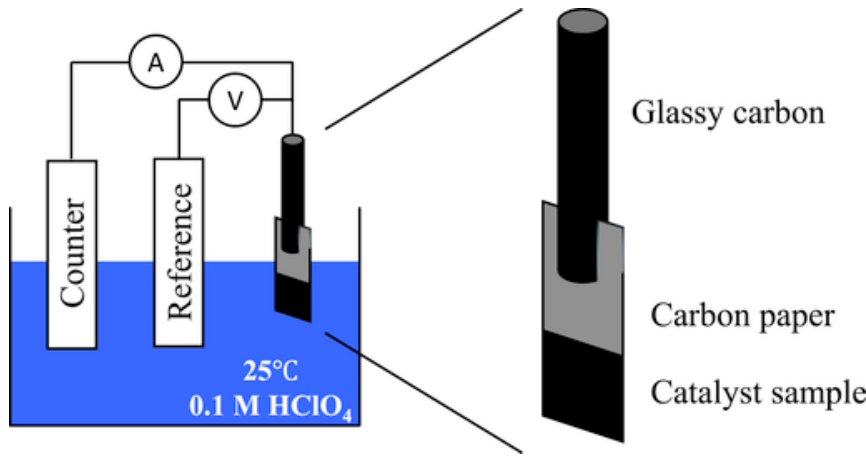

Fig. 1. Schematic of three-electrode cell including a special working electrode designed in this work.

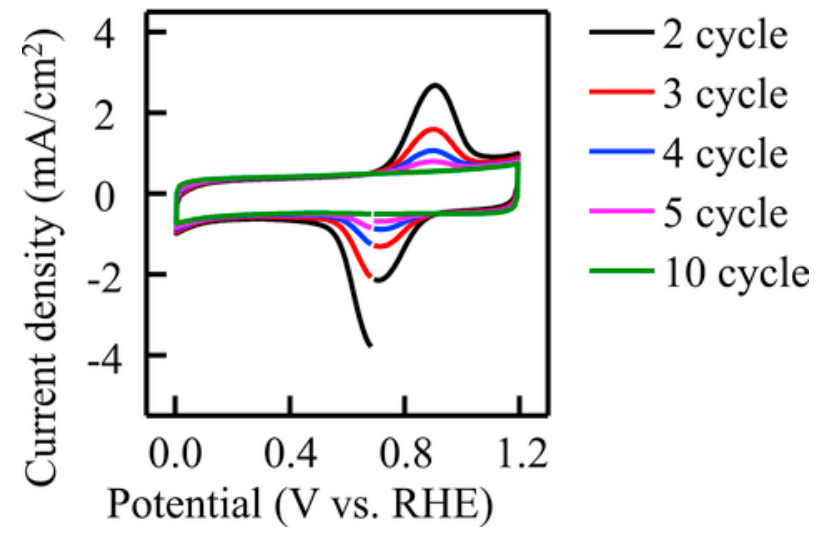

Fig. 2. CV curves of Fe-N-doped CNWs in first few cycles.

The dopings of Fe and $\mathrm{N}$ into CNWs are carried out by Fe sputtering and $\mathrm{N}$ plasma, respectively. The onset potentials corresponding to ORR activities are evaluated from steady-state curve of cyclic voltammogram. The composition and structure are characterized from XPS spectra before and after ORR measurements. The onset potential increases in the order of non-, N- and Fe-N-doped CNW catalysts. The ORR activities for $\mathrm{N}$ - and $\mathrm{Fe}-\mathrm{N}$-doped $\mathrm{CNW}$ catalysts are ascribed to pyridinic $\mathrm{N}$ and $\mathrm{Fe}-\mathrm{N}$, respectively, stabilized near the surface of carbon matrix. Moreover, it is shown that such ORR activities due to pyridinic $\mathrm{N}$ and $\mathrm{Fe}-\mathrm{N}$ near the surface are further promoted by Fe and Fe-N incorporated beneath the surface.

\section{Experimental}

\subsection{Sample preparation}

Non-doped, N-doped, and Fe-N-doped CNWs were prepared by using dc-PECVD/sputtering hybrid system. Non-doped or pure CNWs were synthesized by dc-PECVD. The doping of $\mathrm{N}$ into CNWs was carried out by N-plasma so that N-doped CNW were obtained. For the synthesis of Fe-N-doped CNWs, Fe and $\mathrm{N}$ were doped into CNWs by operating $\mathrm{N}$ plasma and Fe sputtering simultaneously.

CNWs as mother carbon materials were synthesized on a substrate of carbon paper (Mitsubishi Chemical Corporation, Pyrofil ${ }^{\mathrm{TM}}$ GDL) composed of an open mesh of carbon fiber. Note that the carbon paper has been widely used as a gas diffusion layer in PEMFCs. The square carbon paper of $10 \times 10 \mathrm{~cm}^{2}$ as the substrate was set on a glass plate with sample holder in the chamber and covered by a stainless plate with a square hole of $5 \times 5 \mathrm{~cm}^{2}$ at the center. The carbon paper was heated to a temperature of 600 or $700^{\circ} \mathrm{C}$. In the PECVD, the flow rates of $\mathrm{Ar}, \mathrm{CH}_{4}, \mathrm{H}_{2}$ gases were 80, 15, 5 and 70, 10, $20 \mathrm{sccm}$. The vacuum of about $5.0 \times 10^{-3}$ Torr was kept during the PECVD. The plasma current was $70 \mathrm{~A}$. The PECVD synthesis was performed for $6 \mathrm{~h}$. CNWs was synthesized on the uncovered part of $5 \times 5 \mathrm{~cm}^{2}$ of the carbon paper. The other parts of the carbon paper masked were kept as the original paper.

The dopings of Fe and $\mathrm{N}$ into CNWs were carried out by $\mathrm{N}$ plasma and Fe sputtering, respectively. To control the content of the doping, $\mathrm{N}$ plasma was operated with three types of combinations of flow rates of $\mathrm{Ar}, \mathrm{H}_{2}, \mathrm{~N}_{2}$ gases such as $(60,10,40),(60,0,10)$ and $(60,0,40)$ sccm. Fe sputtering was performed with negative voltage of $600 \mathrm{~V}$ applied to the Fe sputtering target. The ratio of the pulse frequency to width, i.e. Duty ratio, was 5 . These dopings were performed at R.T., 300,600 or $700^{\circ} \mathrm{C}$. For the synthesis of Fe-N-doped CNWs, the dopings of $\mathrm{N}$ and $\mathrm{Fe}$ into $\mathrm{CNW}$ are performed by operating $\mathrm{N}$ plasma and Fe sputtering simultaneously. The $\mathrm{Fe}$ and $\mathrm{N}$ doping were performed at R.T. $\sim 600^{\circ} \mathrm{C}$ for $30 \mathrm{~min}$ or $30 \mathrm{~min}$ of $\mathrm{N}$ plasma treatment with $1 \mathrm{~min}$ of Fe sputtering. The Fe sputtering target was covered by stainless mask when Fe sputtering was not needed.

\subsection{Structural characterization}

The size and morphology of non-doped, N-doped, and Fe-N-doped CNWs on carbon fibers were examined by using a field emission scanning electron microscope (FE-SEM) (S-4300, Hitachi). The structure of the catalyst sample was also characterized by a micro-Raman spectroscopy (NRS-3100, JASCO) where the excitation was $532 \mathrm{~nm}$ from cw Nd: $\mathrm{YVO}_{4}$ laser. To evaluate the composition and structure in the samples, X-ray photoelectron spectroscopy (XPS) (PHI5000, ULVAC-PHI, Inc.) was measured by using Al $\mathrm{K} \alpha \mathrm{X}$-ray source $(1486.6 \mathrm{eV})$. To evaluate the existence of inner elements beneath the sample surface, XPS was measured with Ar ion sputtering. The sputtering rate was $8.77 \mathrm{~nm} / \mathrm{min}$ conversion verses $\mathrm{SiO}_{2}$. Energy calibration was established by referring binding energy of $\mathrm{C} 1 \mathrm{~s}$ of common adventitious carbon. Additionally, secondary ion mass spectrometry (TOF-SIMS) (TOF-SIMS5, ION-TOF) was evaluated to define the existence of infinitesimal amount of $\mathrm{Fe}$ and its compounds on ORR measured sample.

\subsection{Electrochemical characterization}

ORR activities for non-doped, N-doped, and Fe-N-doped CNWs were measured in an electrolyte of $0.1 \mathrm{M} \mathrm{HClO}_{4}$ aqueous solution by using a three-electrode cell with a platinum wire as the counter electrode, a reversible hydrogen electrode (RHE) as the reference electrode, and a sample paper inserted into glassy carbon rod as the working electrode as shown in Fig. 1. The potential of the electrodes was controlled by a potentiostat (HZ-5000, HOKUTO DENKO corp.). As shown in Fig. 1, the sample paper is the carbon paper with catalyst of $1 \times 2.5 \mathrm{~cm}^{2}$ cut down as including the deposited catalyst part of $1 \times 1 \mathrm{~cm}^{2}$ and virgin part of $1 \times 1.5 \mathrm{~cm}^{2}$. The virgin part of carbon paper was inserted into the cutting part of one side end of the glassy carbon rod. Only deposited catalyst part of carbon paper was immersed into the electrolyte solution to evaluate ORR activity. Thus, in this work with samples directly synthesized on carbon papers, electrochemical measurement can be carried out without troublesome sample preparations such as mixing with Nafion, etc, which have been generally done for sample preparations in the measurements. These samples with carbon papers, which have been usually used with gas diffusion layer in PEMFCs, also can provide more realistic properties as electrodes in fuel cells. Additionally, in this 
(d) Fe-N-doped $\mathrm{CNW}$

(c) N-doped $\mathrm{CNW}$

(b) non-doped $\mathrm{CNW}$

(a) Carbon paper
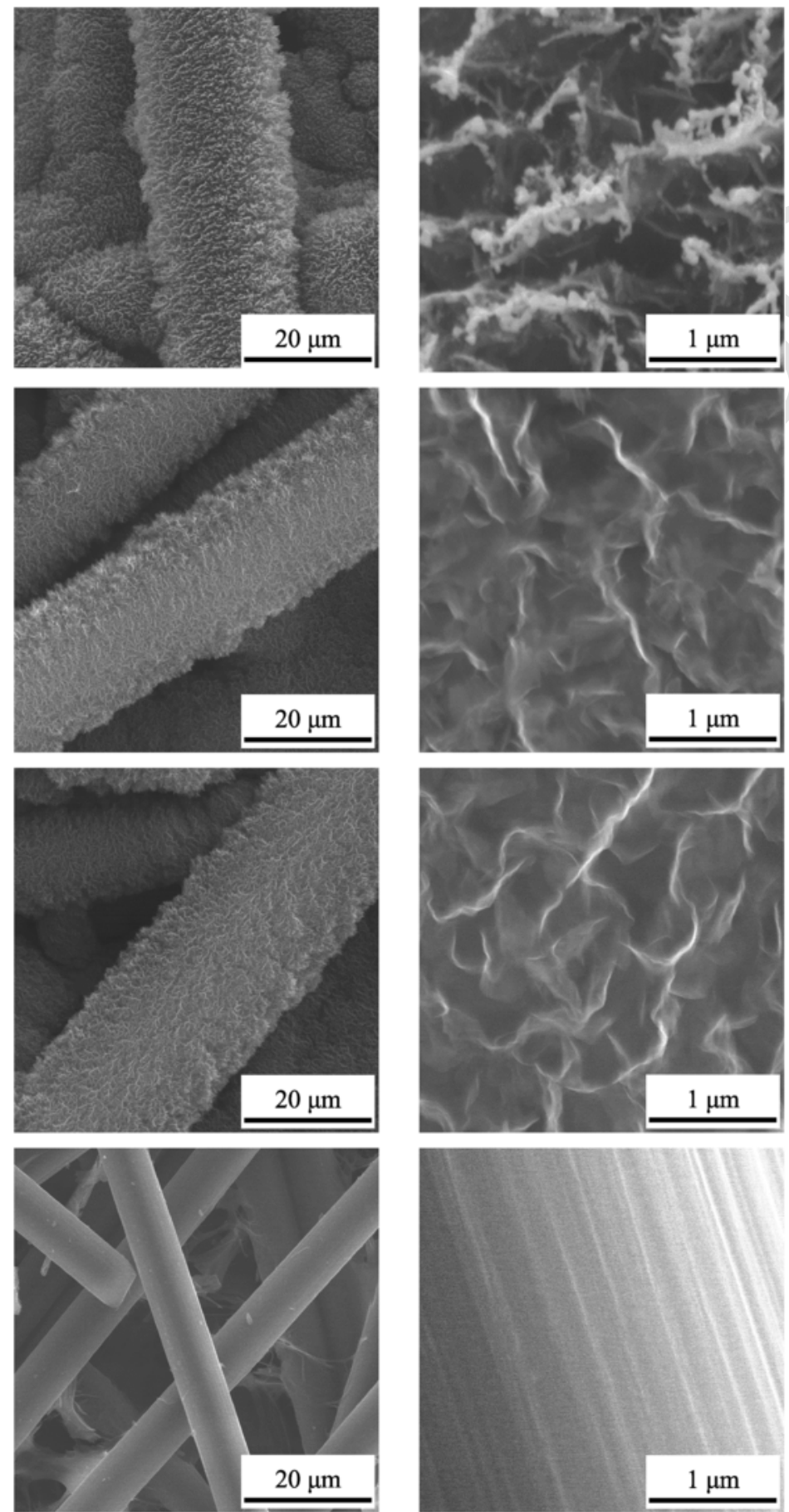

Fig. 3. Typical SEM images of (a) carbon paper and (b) non-doped CNW (c) N-doped CNW (d) Fe-N-doped CNW catalysts.

work with deposited carbon paper itself as electrode, it is easy to characterize the sample paper after the measurements, since the sample paper is easily took out from the working electrode after the measurement.

For activating the electrodes, cyclic voltammograms (CVs) of several 100 cycles for each sample were collected in $\mathrm{N}_{2}$ in the potential range of $0-1.2 \mathrm{~V}$ (vs. RHE) at a scan rate of $100 \mathrm{mV}^{-1}$ at $25^{\circ} \mathrm{C}$ controlled by water bus. Especially, in the $\mathrm{CV}$ curves for samples doped by Fe sputtering, the peaks corresponding to the oxidation and reduction of Fe were clearly observed at $0.9 \mathrm{~V}$ and $0.7 \mathrm{~V}$, respec- tively, as shown Fig. 2. The intensities of both peaks decreased with cyclic measurements. This means that the elution of unstable Fe occurs in acid solution during the measurements. The peaks related to Fe finally disappeared so that steady-state CV curves were obtained usually after about 300 cyclic measurements, i.e. $120 \mathrm{~min}$. Thus, in all measurement, the CV was repeatedly measured about several hundred cycles until a steady-state CV was achieved. After that, CV experiments were carried out at scan rate of $5 \mathrm{mVs}^{-1}$ in the potential range of 1.2 to $0.2 \mathrm{~V}$ in $\mathrm{N}_{2}$ and $\mathrm{O}_{2}$ saturated solution. ORR curve was acquired under oxygen-saturated conditions with subtraction of the 


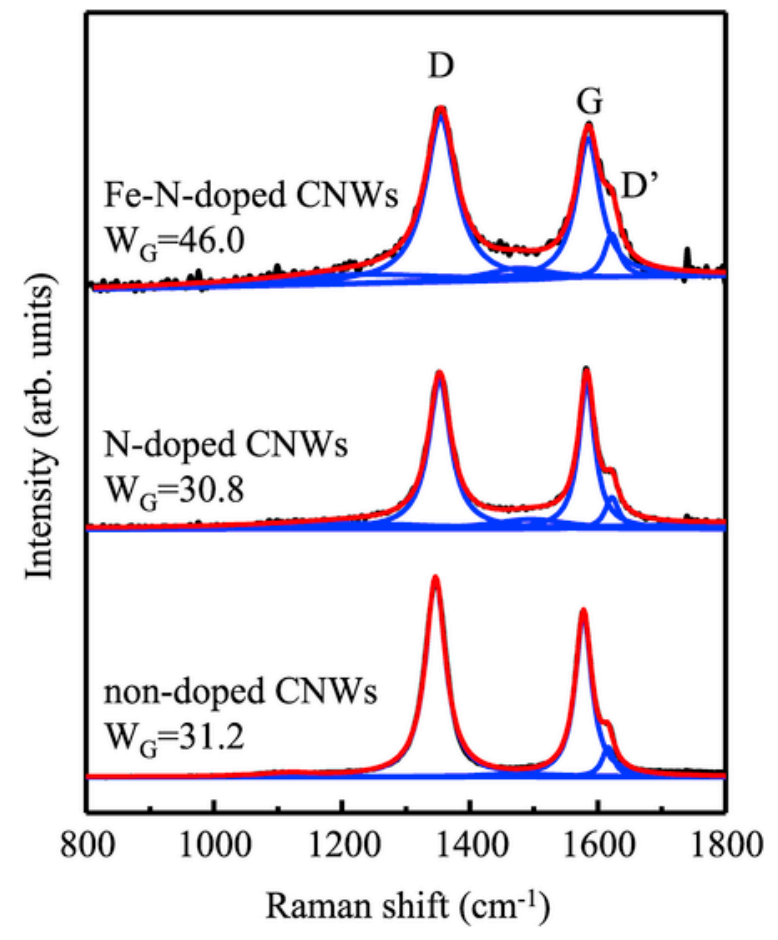

Fig. 4. Typical Raman spectra of non-doped, N-doped, Fe-N-doped CNW samples.

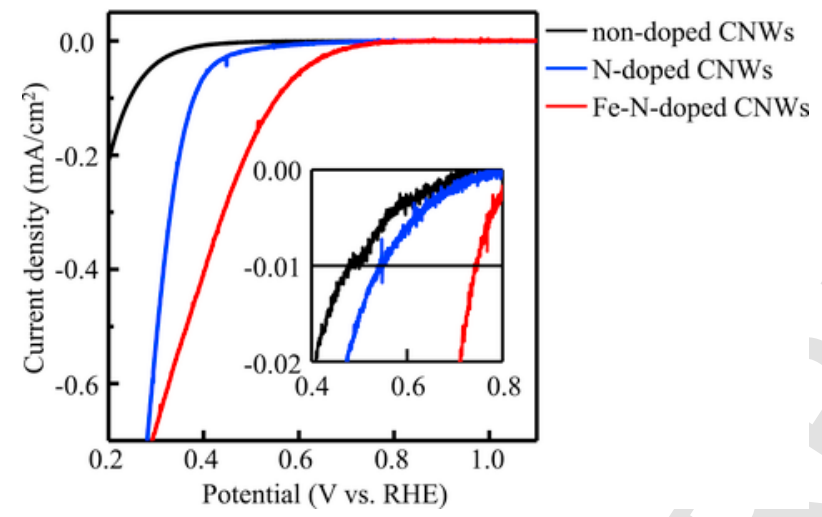

Fig. 5. Typical ORR polarization curves of non-, N-doped, Fe-N-doped CNW samples.

data under nitrogen-saturated conditions as a background, in which the currents were divided by the electrode surface area.

\section{Results and discussion}

\subsection{Morphology and characterization}

Fig. 3 shows typical SEM images of non-doped, N-doped and Fe-N-doped carbon nanowall samples on carbon paper, synthesized by the PECVD/sputtering technique. In all samples, carbon materials are uniformly formed around carbon fibers. The thickness of each carbon material can be estimated by comparing with bare carbon fibers of the diameter of $8 \mu \mathrm{m}$ as shown in Fig. 3(a). The non-doped sample exhibit typical morphology of CNWs as has been reported previously. The thickness is about $9 \mu \mathrm{m}$. No significant change in the morphology is observed in N-doped CNW, although the edges of CNWs are slightly etched by $\mathrm{N}$ plasma, as seen in Fig. 3(c). Fe-N-

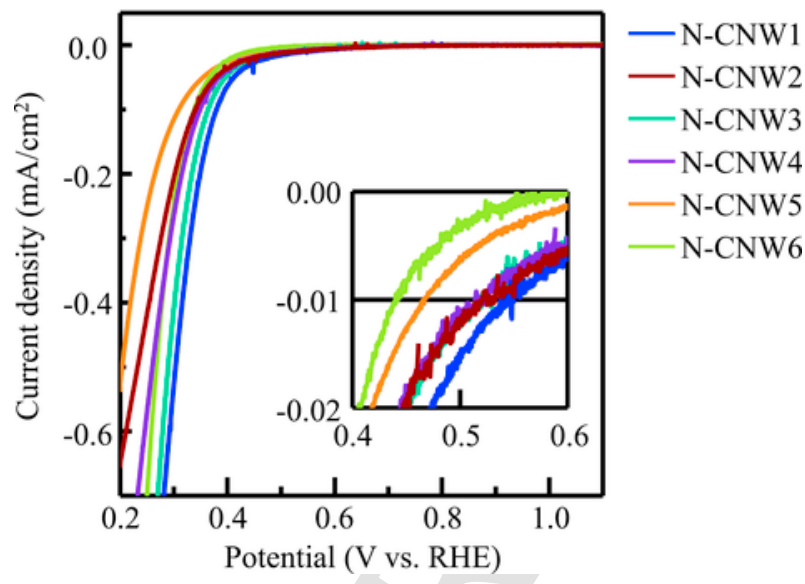

Fig. 6. ORR polarization curves on $\mathrm{N}$-doped $\mathrm{CNW}$ samples with different $\mathrm{N}$ concentrations.

doped CNWs also exhibit similar wall morphology as non-doped CNW as seen in Fig. 3(d). In the extended figure, some small particles of Fe are observed to be deposited on edges and surface of CNWs. These SEM images suggest that doping or deposition of $\mathrm{N}$ and Fe into CNWs occur by $\mathrm{N}$ plasma and Fe sputtering.

The corresponding Raman spectra for non-doped, N-doped, and Fe-N-doped CNW samples are shown in Fig. 4. The Raman spectra were fitted with Lorentzian lines. the Raman spectra exhibit clear $\mathrm{D}$ and $\mathrm{G}$ peaks at 1352 and $1590 \mathrm{~cm}^{-1}$, which can be associated with defect-induced and $E_{2 g}$ modes in graphite, respectively [10]. In non-doped CNWs, peak positions and widths exhibit typical features of CNWs has been reported so far. In N-doped CNW, both peak widths occur slight narrowing, compared with non-doped CNW. This can be ascribed to the etching of amorphous layer covering CNW surface by $\mathrm{N}$ plasma. On the other hand, in Fe-N-doped CNW, both peak widths are larger than those of non-doped CNWs [11]. In addition, a broad peak between $\mathrm{D}$ and $\mathrm{G}$ bands is also appeared at $1513 \mathrm{~cm}^{-1}$, which has not been observed in non-doped CNWs but amorphous-like carbon [12-14]. This means that the degree of graphitization of the Fe-N-doped CNWs is poor compared with that of non-doped CNWs, although the morphology of Fe-N-doped $\mathrm{CNW}$ is similar to that of $\mathrm{CNW}$. The poor graphitization can be attributed to $\mathrm{N}$ plasma and $\mathrm{Fe}$ sputtering.

To confirm the doping of Fe and N, XPS spectra were measured for non-doped, N-doped, and Fe-N-doped CNW as mentioned above. In non-doped CNW sample, as expected, no peak corresponding to Fe and $\mathrm{N}$ is observed in XPS spectrum. On the other hand, in N-doped $\mathrm{CNW}$, clear peak assigned to $\mathrm{N}$ is appeared. From the XPS analysis, the concentration of $\mathrm{N}$ is estimated to be 6.1 at.\%. This means that $\mathrm{N}$ is successfully doped in CNWs by using the plasma of $\mathrm{N}_{2}$. In Fe-N-doped CNW, peaks assigned to Fe and $\mathrm{N}$ are clearly observed. From the XPS analysis, the concentrations of $\mathrm{Fe}$ and $\mathrm{N}$ are evaluated to be 2.5 and 19.8 at. \%, respectively. Thus, both Fe and $\mathrm{N}$ are successfully doped in CNWs by using the plasma of $\mathrm{N}_{2}$ and the sputtering of Fe simultaneously.

\subsection{Electrochemical reaction}

To evaluate the ORR activity for non-doped, N-doped, and Fe-N-doped CNW samples, Cyclic Voltammetry (CV) measurements were carried out by using a three-electrode cell with an electrolyte of $0.1 \mathrm{M} \mathrm{HClO}_{4}$ aqueous solution. Samples with carbon fiber substrates were used as working electrode. In CV measurements, unstable

CV 

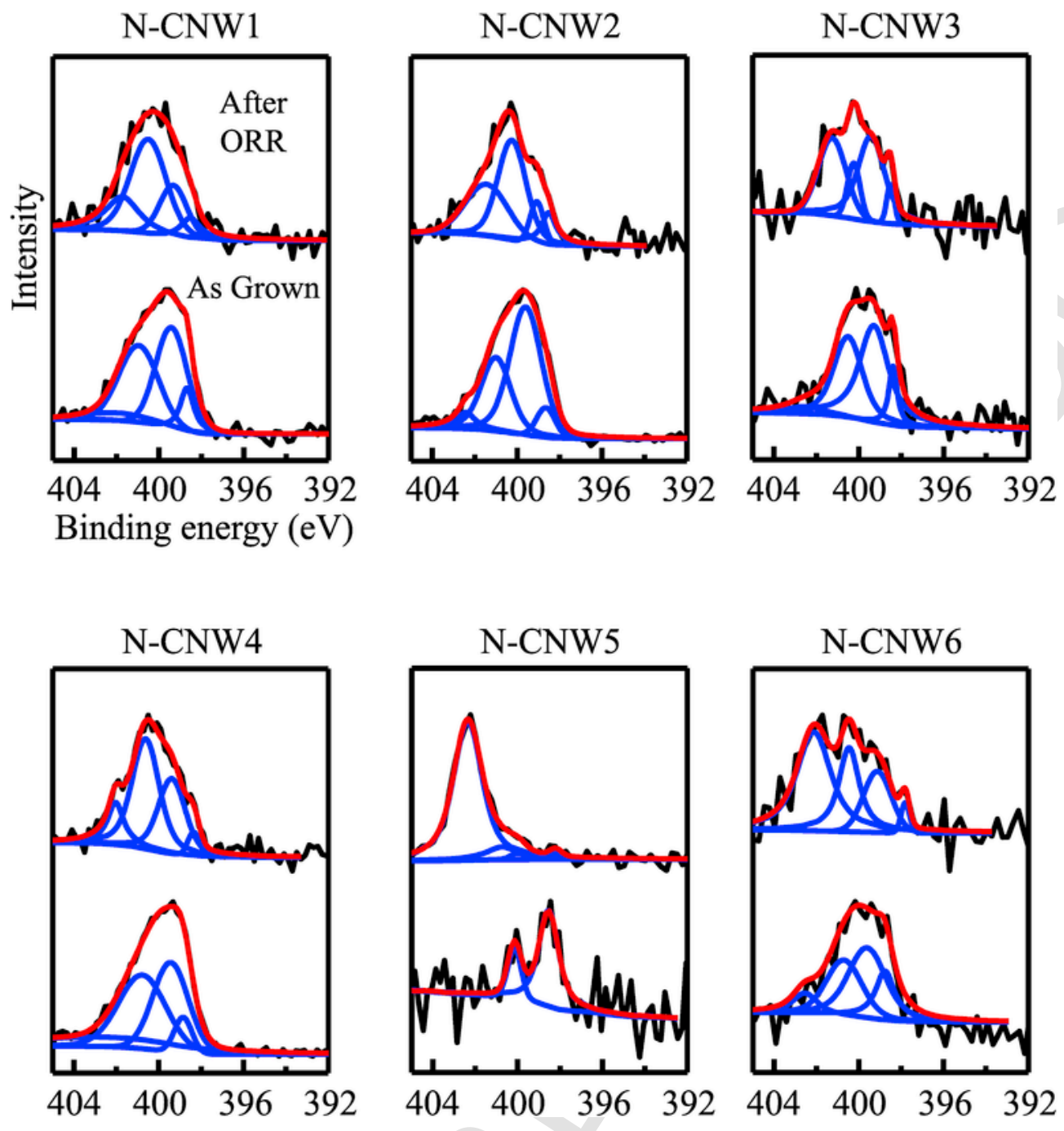

Fig. 7. Extended XPS spectra of N 1s for N-doped CNW samples corresponding to Fig. 4 before and after ORR measurements.

are initially obtained since the elution of unstable species such as $\mathrm{Fe}$ and $\mathrm{N}$ in sample surface occurs in acid solution. Thus, in our experiments, CV was repeatedly measured until a steady-state CV was accomplished. The ORR activity was analyzed from the steady-state CV. Fig. 5 shows current density as a function of potential vs. RHE obtained from the steady-state CVs. The onset potential in the figure corresponds to the ORR activity and is defined as the potential at $-10 \mu \mathrm{A}$ current density in this paper. The onset potentials of typical non-doped, N-doped, and Fe-N-doped CNW samples are 0.39, 0.55, and $0.72 \mathrm{~V}$ vs. RHE. It is obvious that the onset potential corresponding to ORR activity increases in order of non-doped, N-doped, and Fe-N-doped CNW. This order of ORR activity in CNW-based catalysts is in good agreement with that in other carbon-based catalysts has been reported so far [15]. Thus, not only $\mathrm{N}$ but also Fe doping can promote higher ORR activity.

\section{3. $\mathrm{N}-\mathrm{CNWS}$}

To more clarify ORR activity in N-doped CNW, samples with different concentrations of $\mathrm{N}$ were synthesized, and the ORR activities were measured as shown in Fig. 6 . The values of onset potentials corresponding to ORR activities for the samples with various $\mathrm{N}$ concen- trations are higher than $0.39 \mathrm{~V}$ vs. RHE for non-doped or pure CNW samples. This means that N-doping leads to the increase of the onset potential of ORR activity in CNW catalysts. Additionally, it should be noted that the values of onset potentials for N-doped CNW catalysts are limited in the range of $0.40-0.55 \mathrm{~V}$ vs. RHE. The values are in good agreement with those in ORR activities for pyridinic-N doped HOPG reported recently [16]. This can be also understood from the fact that CNWs are composed of graphite domains with high quality, as HOPG [11,17].

XPS spectra after ORR measurements for all N-doped CNW samples are changed compared with those before ORR measurements. This is due to the elution and absorption of some species such as $\mathrm{N}$ in CNW surface by immersing in acid solution and pre-measurement of ORR as mentioned above. This means that XPS before ORR measurement might not reflect active site or real state during ORR. We therefore measured XPS spectra of all samples after ORR measurement, which correspond to steady states associated with active sites in catalysts during ORR. We analyzed XPS spectra before and after ORR measurements. In the XPS analysis, not only total concentration of each elements but also their component concentrations were analyzed. 

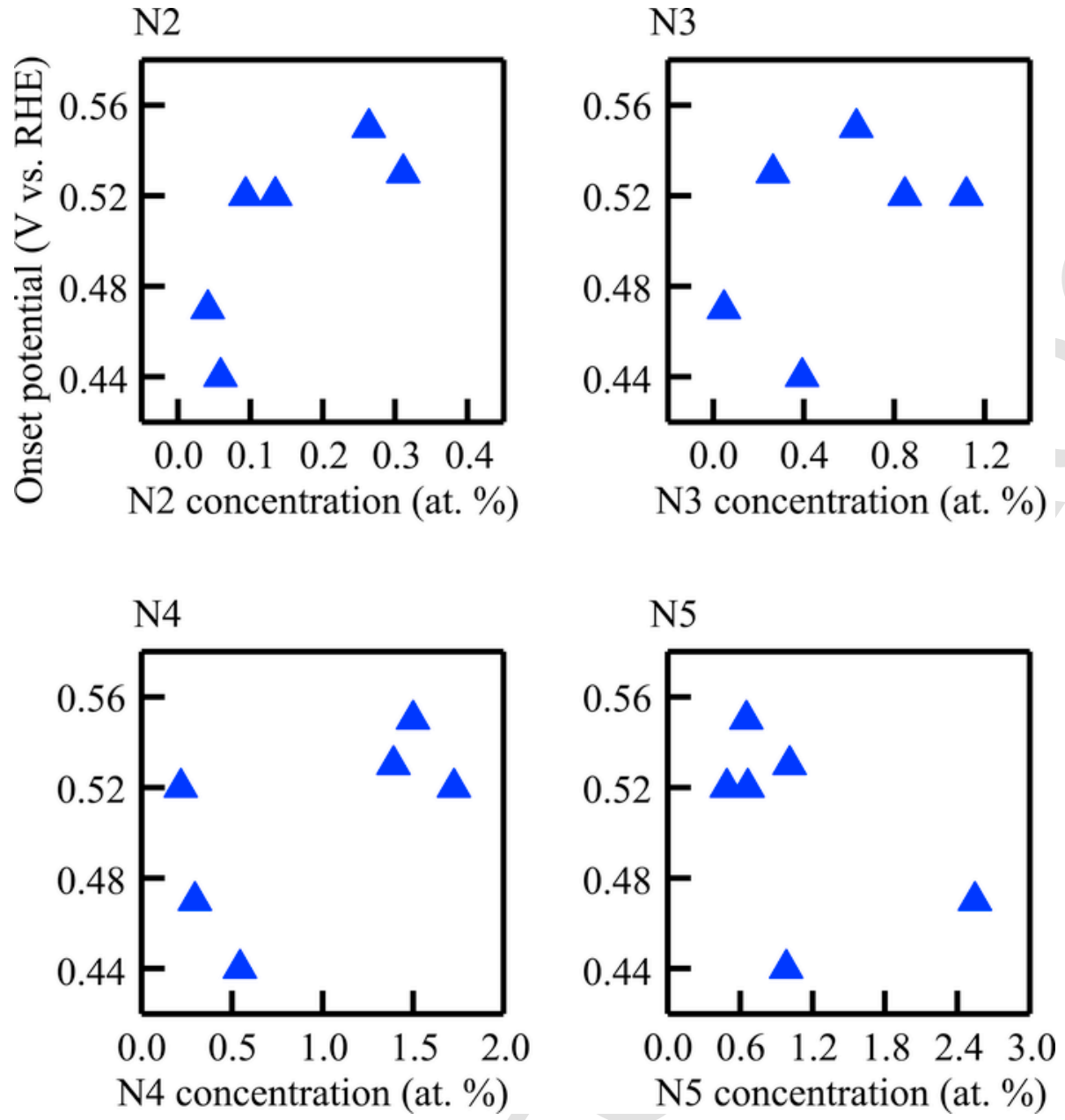

Fig. 8. Relations between onset potentials and concentrations of N components of N2, N3, N4, and N5 for N-doped CNW samples corresponding to Figs. 6 and 7.

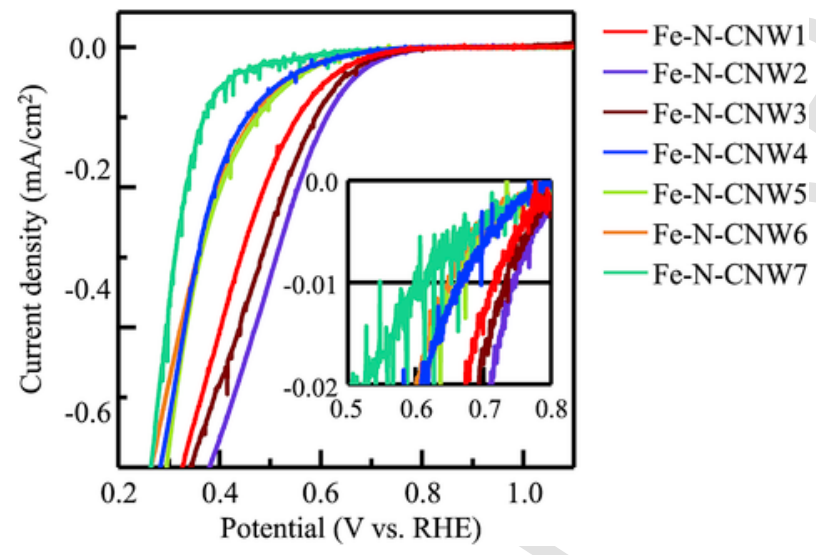

Fig. 9. ORR polarization curves on Fe-N-doped $\mathrm{CNW}$ samples with different $\mathrm{Fe}$ and $\mathrm{N}$ concentrations.

Fig. 7 shows extended XPS spectra of N 1s for various N-doped CNW samples before and after ORR measurement. It is observed that the spectra after ORR are changed compared with those before ORR. The spectra are mainly composed of four peaks. According to previous reports [18], the peaks can be assigned to pyridinic $\mathrm{N}$ at $398.3 \mathrm{eV}(\mathrm{N} 2)$, pyrrolic $\mathrm{N}$ at $400.1 \mathrm{eV}(\mathrm{N} 3)$, graphitic $\mathrm{N}$ at $401.7 \mathrm{eV}$ (N4), and oxidized $\mathrm{N}$ at $404.9 \mathrm{eV}(\mathrm{N} 5)$. Note that $\mathrm{N} 2$ component in (a) $\mathrm{N} 1 \mathrm{~s}$

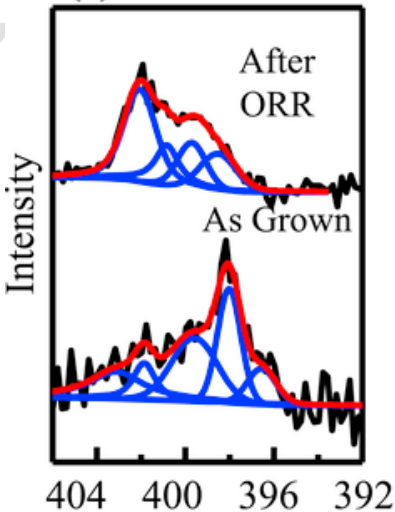

Binding energy $(\mathrm{eV})$ (b) Fe $2 p$

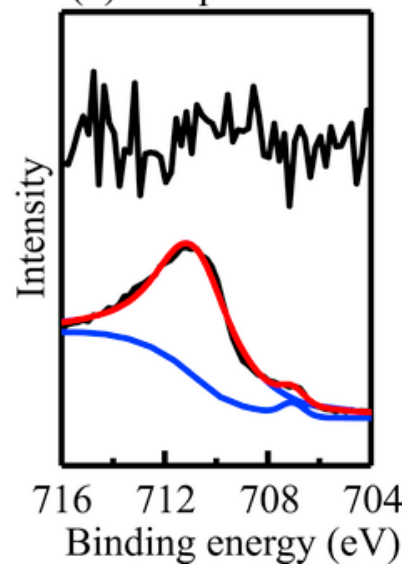

Fig. 10. Extended XPS spectra of (a) $\mathrm{N} 1 \mathrm{~s}$ and (b) Fe 2p for typical Fe-N-doped CNW sample (Fe-N-CNW1) before and after ORR measurement.

$\mathrm{N}$-doped CNW samples without Fe can be assigned to only pyridinic $\mathrm{N}$, although $\mathrm{N} 2$ in $\mathrm{Fe}-\mathrm{N}$-doped $\mathrm{CNW}$ ones should also include a contribution from $\mathrm{N}$ bound to the $\mathrm{Fe}(\mathrm{Fe}-\mathrm{N})$, due to the small difference between binding energies of Fe-N and pyridinic $\mathrm{N}$ [19-21]. The spectra are fitted with four Voigt curves. From the fitting, not only 

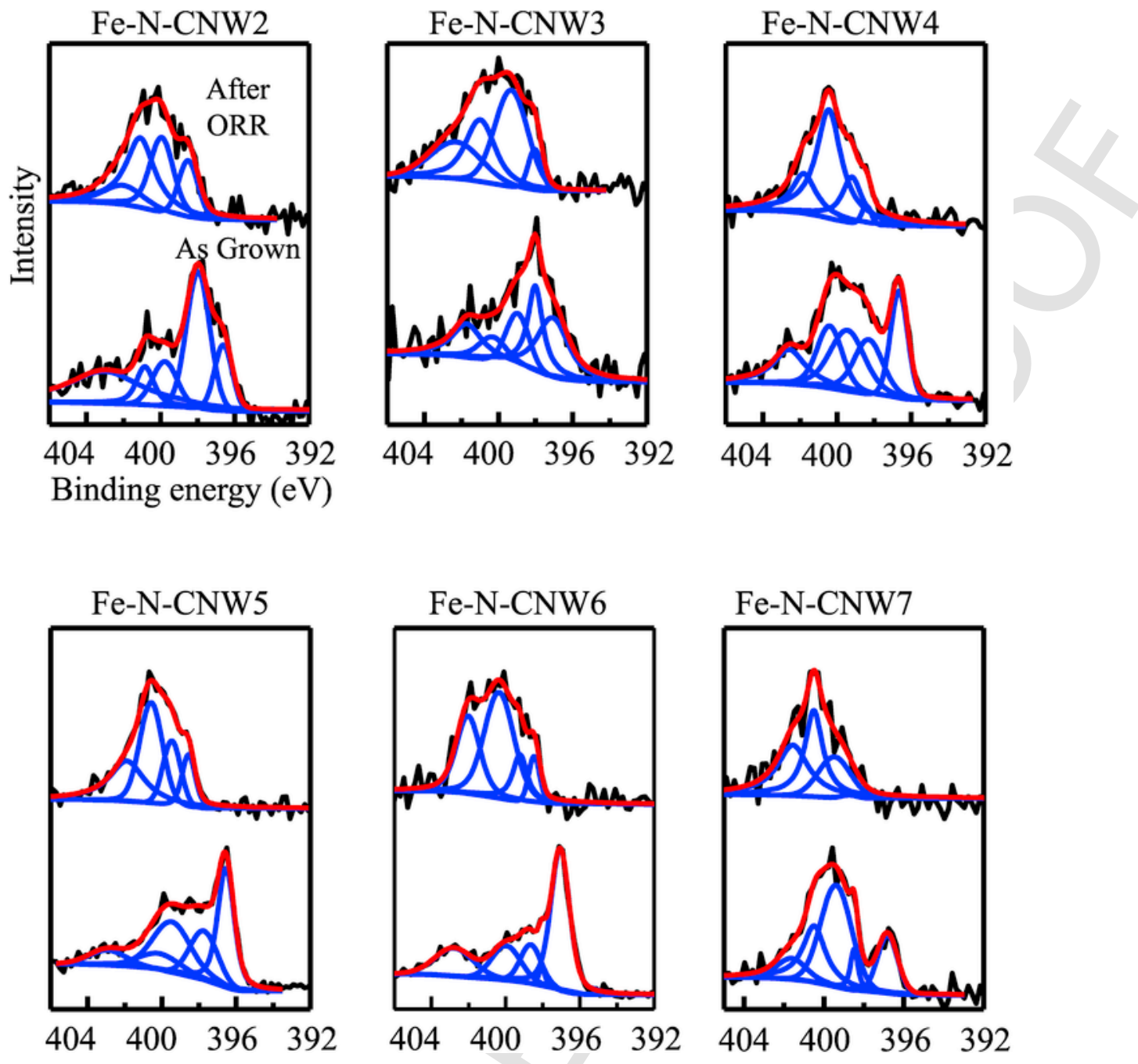

Fig. 11. Extended XPS spectra of N 1s for Fe-N-doped CNW samples corresponding to Fig. 9 before and after ORR measurement.

total concentration of $\mathrm{N}$ but also the component concentrations are analyzed. From the analysis, it is found that only $\mathrm{N}$ component corresponding to pyridinic $\mathrm{N}$ (N2) after ORR measurement is strongly correlated to the onset potential corresponding to ORR activity for N-doped CNW samples as shown in Fig. 8, although the intensity of $\mathrm{N} 2$ is not high compared with those of other components. Namely, the onset potential increases with the increasing of N2 concentration as seen in Fig. 8. Other components are not absolutely correlated with ORR activity. This means that the pyridinic $\mathrm{N}$ corresponding to N2 plays a crucial role for ORR activity. This is also in good agreement with that in ORR activity for pyridinic N doped HOPG reported recently [16].

\section{4. $\mathrm{Fe}-\mathrm{N}-\mathrm{CNW}$}

Furthermore, to clarity ORR activity in Fe-N-doped CNW catalysts, samples with different concentrations of $\mathrm{Fe}$ and $\mathrm{N}$ are synthesized, and the ORR activities were measured as shown in Fig. 9. Interestingly, the values of the onset potentials in the samples are ranged from 0.61 to $0.75 \mathrm{~V}$ vs. RHE. These values are clearly higher than even a maximum value of $0.55 \mathrm{~V}$ in $\mathrm{N}$-doped $\mathrm{CNW}$ catalysts as mentioned above. This means that higher ORR activity needs not only $\mathrm{N}$ doping but also Fe doping.
Compared with that in N-doped CNW samples, significant change in XPS spectra was observed after ORR measurements for all Fe-N-doped CNW samples. This is obviously ascribed to the significant elution of unstable species such as Fe in the surface of Fe-N-doped CNW samples by immersing in acid solution and pre-measurements of ORR, as mentioned above. This is probably related to the elution of defective and unstable structure in the surface of carbon matrix deposited by Fe and N. This means that XPS spectra of the samples before ORR measurements might not reflect active sites or real states during ORR. Therefore, we also measured XPS spectra after ORR measurement for Fe-N-doped CNW samples, which correspond to steady states associated with the active sites in catalysts during ORR. We analyzed XPS spectra before and after ORR measurements. In the XPS analysis, not only total concentration of each elements but also their component concentrations were also analyzed.

As mentioned above, XPS spectra after ORR measurements for all Fe-N-doped CNW samples are drastically changed compared with those before ORR. Especially, it should be noted that Fe $2 p$ peaks for all of samples are drastically reduced and disappeared after ORR measurement as shown Fig. 10(b). On the other hand, N 1s is clearly observed even after ORR measurement as shown in Fig. 10(a). However, as mentioned above, the onset potentials in Fe-N-doped CNW samples are higher than those of $\mathrm{N}$-doped $\mathrm{CNW}$ ones without $\mathrm{Fe}$, in 

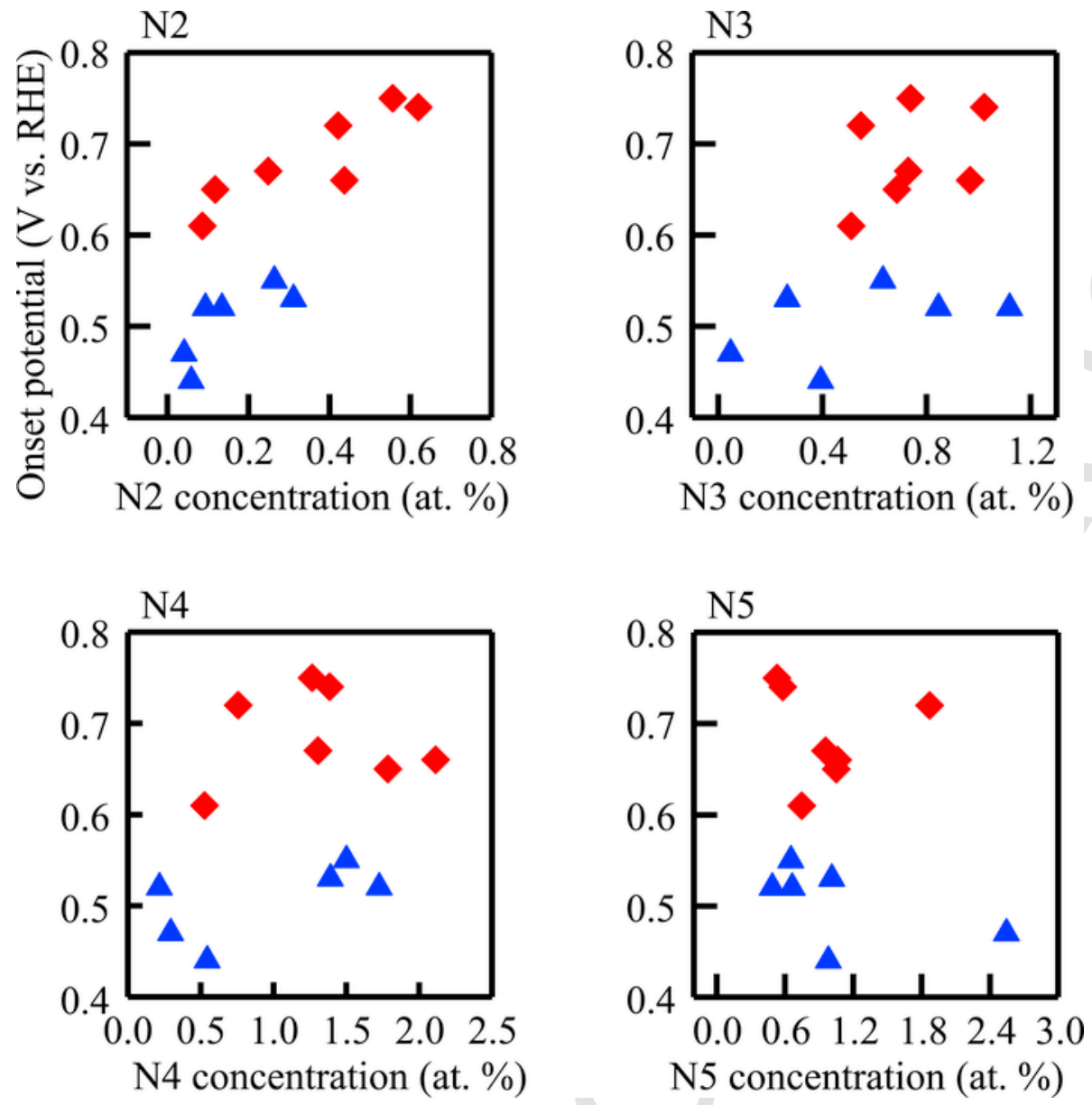

Fig. 12. Relations between onset potentials and concentrations of N components of N2, N3, N4, and N5 for Fe-N-doped CNW samples corresponding to Figs. 9-11.

(a) $\mathrm{N} 1 \mathrm{~s}$

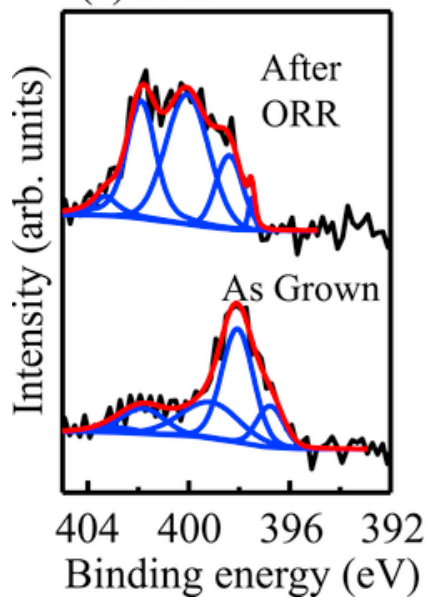

(b) Fe 2p

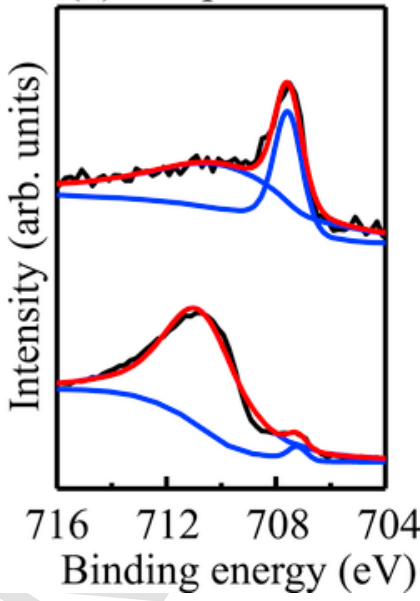

Fig. 13. Typical extended XPS spectra of (a) N 1s and (b) Fe 2p for N-doped Fe-incorporating CNW catalysts before and after ORR measurements.

spite of the fact that no Fe in Fe-N-doped CNW ones after ORR measurements is detected by XPS. It is therefore suggested that trace Fe below the detection limit ( $\sim 0.1$ at. $\%)$ of XPS might lead to higher onset potentials of ORR activities than those of N-doped CNW catalysts.

To confirm the trace Fe, TOF-SIMS was measured for Fe-N-doped CNW samples after ORR measurement [22]. As a result, trace Fe and $\mathrm{Fe}$ compounds were clearly detected for the samples even after ORR measurement. In the particular TOF-SIMS analysis, Fe concentration, which is equivalent to that in XPS, is estimated by using $\mathrm{Fe}^{+}$signal intensity. For example, for Fe-N-CNW1 sample, the $\mathrm{Fe}^{+}$intensity of as-grown sample is assumed as 20 at. \% of Fe, which is obtained by XPS. According to the TOF-SIMS analysis, the total concentrations of Fe in the sample after ORR measurement is estimated to be 0.05 at. $\%$ for Fe-N-CNW1. The low concentration is a reason why Fe concentration in samples after ORR could not be detected by XPS. Additionally, Fe-N and Fe-N-C compounds are also detected by TOF-SIMS, although it is difficult to estimate such compound's concentrations. It is therefore suggested that such trace Fe and Fe compounds promote ORR activity. Such promotion of ORR activity by trace Fe is also supported by previous report [23-26].

The extended XPS spectra of $\mathrm{N}$ 1s for various Fe-N-doped CNW samples before and after ORR measurement are shown in Fig. 11. It is observed that the spectra after ORR are drastically changed compared with those before ORR. The spectra are composed of five peaks. According to previous reports [19-21]. these peaks can be assigned to iron nitrides at 396.4 (N1), pyridinic $\mathrm{N}$ and Fe-N at $398.3 \mathrm{eV}(\mathrm{N} 2)$, pyrrolic $\mathrm{N}$ at $400.1 \mathrm{eV}(\mathrm{N} 3)$, graphitic $\mathrm{N}$ at $401.7 \mathrm{eV}(\mathrm{N} 4)$, and $\mathrm{Ox}-$ idized $\mathrm{N}$ at $404.9 \mathrm{eV}$ (N5). Note that N2 component in Fe-N-doped $\mathrm{CNW}$ samples should include a contribution from $\mathrm{N}$ bound to the $\mathrm{Fe}(\mathrm{Fe}-\mathrm{N})$, due to the small difference between binding energies of $\mathrm{Fe}-\mathrm{N}$ and pyridinic $\mathrm{N}$. The spectra are fitted with five 


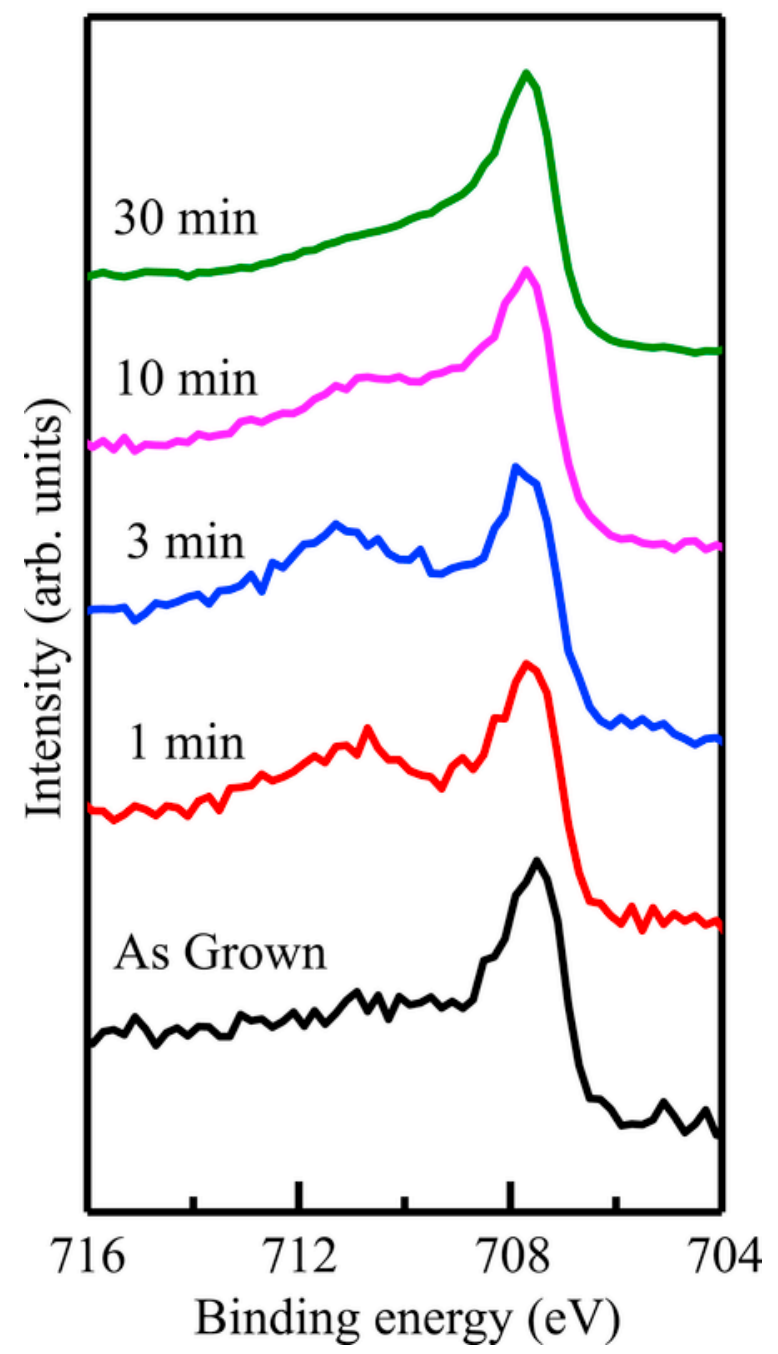

Fig. 14. Typical etching time dependence of XPS spectrum of Fe $2 p$ for N-doped Fe-incorporating $\mathrm{CNW}$ samples corresponding to Fig. 13. The etching is carried out by sputtering with Ar ion. (a) $\mathrm{N} 1 \mathrm{~s}$

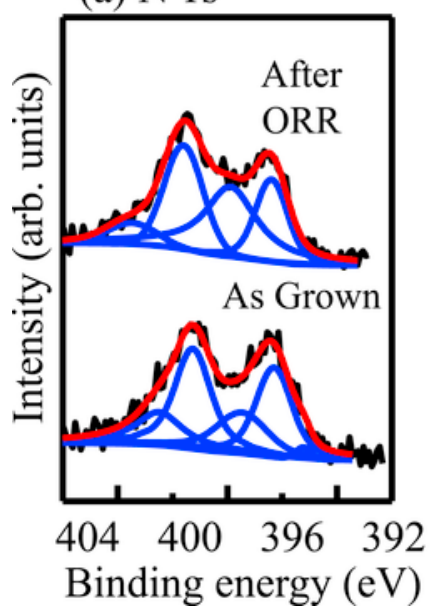

(b) Fe 2p

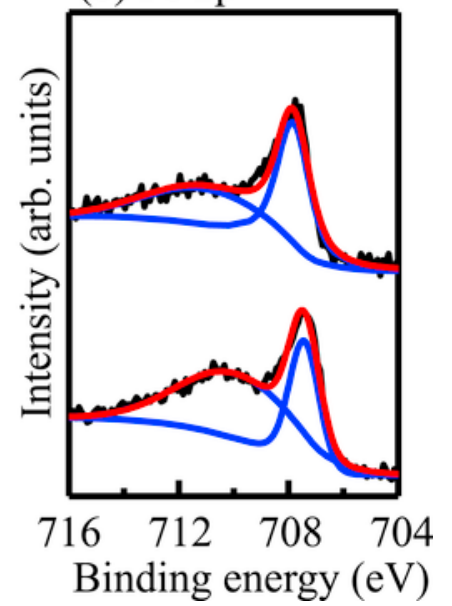

Fig. 15. Typical extended XPS spectra of (a) N 1s and (b) Fe 2p for Fe-N-incorporating carbon catalysts before and after ORR measurements.
Voight curves. From the fitting, not only total concentration of $\mathrm{N}$ but also the component concentration is analyzed.

The peak profile in Fe-N-doped CNWs before ORR are different from those of N-doped CNW. Namely, N2 peak is predominant in some of Fe-N-doped CNWs. This means that pyridinic $\mathrm{N}$ and $\mathrm{Fe}-\mathrm{N}$ corresponding to the N2 is preferentially formed. However, the N2 peak intensity is drastically reduced after ORR. Thus, it is suggested that the reduction of $\mathrm{N} 2$ peak might be mainly ascribed to the elution of $\mathrm{Fe}$ as mentioned above. This is probably related to the elution of defective and unstable structure in the surface of carbon matrix deposited by $\mathrm{Fe}$ and $\mathrm{N}$.

From XPS component analysis in light of ORR activity, it is found that only $\mathrm{N} 2$ component corresponding to pyridinic $\mathrm{N}$ and Fe-N after ORR measurement is strongly correlated to the onset potential for Fe-N-doped CNW samples as shown in Fig. 12. Namely, the onset potential increases with the increasing of $\mathrm{N} 2$ concentration, as $\mathrm{N}$-doped CNWs catalysts. On the other hand, other $\mathrm{N}$ components are not absolutely correlated with ORR activity. This suggests that pyridinic $\mathrm{N}$ and/or Fe-N corresponding to N2 plays a crucial role for ORR activity, as N-CNWs. However, ORR activities in Fe-N-doped CNW samples are clearly higher than those in N-doped ones even in the same concentration of $\mathrm{N} 2$, as mentioned above. Thus, it is suggested that $\mathrm{Fe}-\mathrm{N}$ rather than pyridinic $\mathrm{N}$ corresponding to $\mathrm{N} 2$ contributes to higher ORR activities.

For example, for Fe-N-CNW1 sample, N2 concentration is estimated to be 0.42 at. $\%$ by XPS analysis as mentioned above. The concentration is quite large compared with 0.05 at. \% of Fe detected by using TOF-SIMS. This means that N2 component observed in XPS is mainly attributed to pyridinic $\mathrm{N}$ rather than Fe-N. However minor $\mathrm{Fe}-\mathrm{N}$ in $\mathrm{N} 2$ component contributes to the higher ORR activity in Fe-N-CNW samples. Additionally, the strong correlation is observed between $\mathrm{N} 2$ and higher ORR activity. Thus, it is suggested that the concentration of $\mathrm{Fe}-\mathrm{N}$ is correlated with that of $\mathrm{N} 2$ component, although the concentration is much lower than that of pyridinic $\mathrm{N}$ in Fe-N-doped CNW catalysts.

Moreover, it is found that high concentration of $\mathrm{N} 2$ is formed in $\mathrm{Fe}-\mathrm{N}$-doped $\mathrm{CNW}$, compared with that in $\mathrm{N}$-doped $\mathrm{CNW}$ as seen in Fig. 12. This means that more pyridinic $\mathrm{N}$ species are stabilized by the doping of $\mathrm{Fe}$. The stabilization of pyridinic $\mathrm{N}$ can occur by the formation of Fe-N. These results suggest the role of Fe not only directly participating in active site as $\mathrm{Fe}-\mathrm{N}$ but also promoting the formation of stable pyridinic $\mathrm{N}$.

As mentioned above, in Fe-N-CNWs, Fe and $\mathrm{N}$ are deposited on the surface of CNWs. Most of the deposited Fe species can be eluted in acid solution and during pre-measurement of ORR. This is due to the elution of defective and unstable structure in the surface of carbon matrix deposited by $\mathrm{Fe}$ and $\mathrm{N}$. As a result, it was shown that residual stable Fe-N on the surface of CNWs leads to higher ORR activity.

To further examine the effect of Fe, or metallic Fe, inside CNWs, Fe species incorporated inside CNWs were prepared by the sputtering of Fe with carbon deposition by PECVD. The morphology of typical CNWs is kept even in Fe-incorporating CNWs. For the Fe-incorporating CNWs, $\mathrm{N}$ doping was carried out by $\mathrm{N}$ plasma treatment. As a result, $\mathrm{N}$-doped Fe-incorporating CNWs were obtained. For these samples, XPS peak of Fe $2 p$ is clearly observed even after ORR measurement as shown in Fig. 13(b), in contrast with the disappearance of Fe peak in Fe-N-CNWs as mentioned above. The content of residual $\mathrm{Fe}$ is 0.58 at. \%, whereas as-grown one includes $\mathrm{Fe}$ content of 0.82 at. $\%$. Therefore, $\sim 70 \%$ of Fe doped in CNWs is kept even after ORR measurements. Two main peaks observed at $\sim 708 \mathrm{eV}$ and $711 \mathrm{eV}$ are associated with metallic $\mathrm{Fe}$ and $\mathrm{Fe}^{2+}, \mathrm{Fe}^{3+}$ oxidation state or Fe-N, respectively. From the relative intensity, it is found that 
metallic $\mathrm{Fe}$ is predominant in the samples after ORR. This means that most of metallic Fe is stably doped in N-doped Fe-incorporating CNWs.

Additionally, the XPS peak of metallic Fe at $\sim 708 \mathrm{eV}$ is also observed even inside the samples as shown in Fig. 14, where the measurements are carried out by etching the sample surface by sputtering with Ar ion. This means that the stable metallic Fe species are contained inside the samples. Additionally, N2 peak is also clearly observed after ORR measurement. It should be noted that the onset potential exhibits $0.8 \mathrm{~V}$ that is higher than those due to $\mathrm{Fe}-\mathrm{N}$ in Fe-N-CNWs. However, only metallic Fe exhibits no significant ORR activity [9]. Thus, it is suggested that $\mathrm{Fe}$, or metallic $\mathrm{Fe}$, inside the samples assists the ORR activity due to Fe-N on the surface, although it is not active site. Namely, metallic Fe inside the samples gives rise to synergy effect for the ORR activity with Fe-N on the surface.

Furthermore, both $\mathrm{Fe}$ and $\mathrm{N}$ species incorporated inside carbon matrix were prepared. In the synthesis, $\mathrm{Fe}$ sputtering and $\mathrm{N}$ plasma were simultaneously applied with carbon deposition by PECVD. The morphologies of carbon catalysts incorporating both $\mathrm{Fe}$ and $\mathrm{N}$ species synthesized by this method are often different from that of typical CNWs. It should be noted that further higher onset potentials are observed. The maximum value is over $0.89 \mathrm{~V}$ vs. RHE that is further high compared with those of $\mathrm{N}$-doped Fe-incorporating CNWs. According to XPS of Fe and N, N2 and Fe components are clearly observed even after ORR measurements as shown in Fig. 15. The content of residual $\mathrm{Fe}$ is 0.82 at $\%$, which is larger than 0.32 at \% of as-grown one. Such increase of Fe content after ORR measurements can be related to the elution of unstable $\mathrm{N}$. The existence of $\mathrm{Fe}$ inside the samples are also observed in XPS measurements with etching sample surface by sputtering with Ar ion. These results suggest that $\mathrm{Fe}$ and $\mathrm{Fe}-\mathrm{N}$ inside the samples further promote ORR activity associated with the surface Fe-N. Namely Fe and Fe-N inside the samples give rise to synergy effect on ORR activity of surface Fe-N, although the detailed mechanism is not yet clear.

\section{Conclusion}

We have showed the effect of Fe and N in CNW catalysts on ORR. The onset potential corresponding to ORR activity increases in the order of non-, N- and Fe-N--doped CNW catalysts. The onset potential increases in the order of non-, $\mathrm{N}$ - and Fe-N-doped CNW catalysts. The ORR activity for N-doped CNW catalysts is ascribed to pyridinic $\mathrm{N}$ stabilized near the surface of carbon matrix. The higher ORR activity for Fe-N-doped $\mathrm{CNW}$ catalysts is attributed to trace $\mathrm{Fe}-\mathrm{N}$ rather than pyridinic $\mathrm{N}$ near the surface. It is shown that such ORR activities due to pyridinic $\mathrm{N}$ and $\mathrm{Fe}-\mathrm{N}$ near the surface are further promoted by $\mathrm{Fe}$ and $\mathrm{Fe}-\mathrm{N}$ incorporated beneath the surface. These knowledges provide effective strategy for the synthesis of Fe-N-doped carbon catalysts with higher ORR activities.

\section{Acknowledgement}

This study was supported by Iketani Science and Technology Foundation (0291078-A) and JSPS KAKENHI (17K06797).

\section{References}

[1] Y. Nie, L. Li, Z. Wei, Recent advancements in Pt and Pt-free catalysts for oxygen reduction reaction, Chem. Soc. Rev. 44 (2015) 2168.

[2] C. Tang, Q. Zhang, Nanocarbon for oxygen reduction electrocatalysis: dopants, edges, and defects, Adv. Mater. 29 (2017) 1604103.

[3] S.N. Bhange, S.M. Unni, S. Kurungot, Graphene with Fe and S coordinated active centers: an active competitor for the $\mathrm{Fe}-\mathrm{N}-\mathrm{C}$ active center for oxygen re- duction reaction in acidic and basic $\mathrm{pH}$ conditions, Applied Energy Materials 1 (2018) 368-376.

[4] L. Osmieri, A.H.A.M. Viadela, M. Armandi, S. Specchia, Influence of different transition metals on the properties of $\mathrm{MeeNeC}(\mathrm{Me} 1 / 4 \mathrm{Fe}, \mathrm{Co}, \mathrm{Cu}, \mathrm{Zn})$ catalysts synthesized using SBA-15 as tubular nano-silica reactor for oxygen reduction reaction, Science Direct 41 (2016) 22570-22588.

[5] C.H. Choi, W.S. Choi, O. Kasian, A.K. Mechler, M.T. Sougrati, S. Brügller, K. Strickland, Q. Jia, S. Mukerjee, K.J.J. Mayrhofer, F. Jaouen, Unraveling the nature of sites active toward hydrogen peroxide reduction in $\mathrm{Fe}-\mathrm{N}-\mathrm{C}$ catalysts, Angew. Chem. 56 (30) (2017) 8935-8938.

[6] X. Xu, C. Shi, Q. Li, R. Chen, Fe-N-Doped carbon foam nanosheets with embedded $\mathrm{Fe}_{2} \mathrm{O}_{3}$ nanoparticles for highly efficient oxygen reduction in both alkaline and acidic media, RSC Adv. 7 (2017) 14382-14388.

[7] K. Strickland, E. Miner, Q. Jia, U. Tylus, N. Ramaswamy, W. Liang, M.-T. Sougrati, F. Jaouen, S. Mukerjee, Highly active oxygen reduction non-platinum group metal electrocatalyst without direct metal-nitrogen coordination, Nat. Commun. 6 (2015) 7343.

[8] A. Zitolo, V. Goellner, V. Armel, M.T. Sougrati, T. Mineva, L. Stievano, E. Fonda, F. Jaouen, Identification of catalytic sites for oxygen reduction in ironand nitrogen-doped graphene materials, Nat. Mater. 14 (2015) 937-3942.

[9] K. Hotozuka, R. Yoshie, H. Murata, A. Tateno, G. Ito, N. Kawaguchi, T. Matsuo, H. Ito, I. Kinoshita, M. Tachibana, Fe-N-doped carbon catalysts prepared by hybrid PECVD/sputtering system for oxygen reduction reaction, Chem. Phys. Lett. 679 (2017) 70-76.

[10] A. Jorio, R. Saito, G. Dresselhaus, M.S. Dresselhaus, Raman Spectroscopy in Graphene Related Systems, Wiley-VCH, Berlin, 2011.

[11] S. Kurita, A. Yoshimura, H. Kawamoto, T. Uchida, K. Kojima, M. Tachibana, P. Molina-Morales, H. Nakai, Raman spectra of carbon nanowalls grown by plasma-enhanced chemical vapor deposition, J. Appl. Phys. 97 (2005) 104320.

[12] A. Cuesta, P. Dhamelincourt, J. Laureyns, A. Martínez-Alonso, J.M.D. Tascón, Raman microprobe studies on carbon materials, Carbon 32 (8) (1994) $1523-1532$.

[13] T. Jawhari, A. Roid, J. Casado, Raman spectroscopic characterization of some commercially available carbon black materials: spectral analysis and structural information, Carbon 33 (11) (1995) 1561-1565.

[14] A. Sadezky. H. Muckenhuber, H. Grothe, R. Niessner, U. Pöschl, Raman microspectroscopy of soot and related carbonaceous materials, Carbon 43 (8) 1731-1742.

[15] Z. Liu, J. Yu, X. Li, L. Zhang, D. Luo, X. Liu, X. Liu, S. Liu, H. Feng, G. Wu, P. Guo, H. Li, Z. Wang, X.S. Zhao, Facile synthesis of N-doped carbon layer encapsulated $\mathrm{Fe}_{2} \mathrm{~N}$ as an efficient catalyst for oxygen reduction reaction, Carbon 127 (2018) 636-642.

[16] D. Guo, R. Shibuya, C. Akiba, S. Saji, T. Kondo, J. Nakamura, Active sites of nitrogen-doped carbon materials for oxygen reduction reaction clarified using model catalysts, Science 351 (6271) (2016) 361-365.

[17] K. Kobayashi, M. Tanimura, H. Nakai, A. Yoshimura, H. Yoshimura, K. Kojima, M. Tachibana, Nanographite domains in carbon nanowalls, J. Appl. Phys. 101 (2007), 094306.

[18] E.R. Piñero, D.C. Amorós, A.L. Solano, J. Find, U. Wild, R. Schlögl, Structura characterization of $\mathrm{N}$-containing activated carbon fibers prepared from a low softening point petroleum pitch and a melamine resin, Carbon 40 (2002) 597-608.

[19] H.-W. Liang, W. Wei, Z.-S. Wu, X. Feng, K. Mullen, metal-nitrogen-doped carbon EleCarbon electrocatalysts for highly efficient oxygen reduction reaction, J. Am. Chem. Soc. 135 (43) (2013) 16002-16005.

[20] G. Wu, C.M. Johnston, N.H. Mack, K. Artyushkova, M. Ferrandon, M. Nelson, J.S.L. Pacheco, S.D. Conradson, K.L. More, D.J. Myers, P. Zelenay, Synthesis-structure-performance correlation for polyaniline-Me-C non-precious metal cathode catalysts for oxygen reduction in fuel cells, J. Mater. Chem. 21 (2011) 11392.

[21] H.R. Byon, J. Suntivich, Y.S. Horn, Graphene-based non-noble-metal catalysts for oxygen reduction reaction in acid, Chem. Mater. 23 (2011) 3421-3428.

[22] T. Palaniselvam, V. Kashyap, S.N. Bhange, J.-B. Baek, S. Kurungot, Nanoporous graphene enriched with $\mathrm{Fe} / \mathrm{Co}-\mathrm{N}$ active sites as a promising oxygen reduction electrocatalyst for anion exchange membrane fuel cells, Adv. Funct. Mater. 26 (13) (2016) 2150-2162.

[23] W. Niu, L. Li, X. Liu, N. Wang, J. Liu, W. Zhou, Z. Tang, S. Chen, Mesoporous $\mathrm{N}$-doped carbons prepared with thermally removable nanoparticle templates: an efficient electrocatalyst for oxygen reduction reaction, J. Am. Chem. Soc. 137 (2015) 5555-5562.

[24] J. Liu, X. Sun, P. Song, Y. Zhang, W. Xing, W. Xu, High-Performance oxygen reduction electrocatalysts based on cheap carbon black, nitrogen, and trace iron, Adv. Mater. 25 (2013) 6879-6883.

[25] J. Masa, A. Zhao, W. Xia, Z. Sun, B. Mei, M. Muhler, W. Schuhmann, Trace metal residues promote the activity of supposedly metal-free nitrogen-modified carbon catalysts for the oxygen reduction reaction, Electrochem. Commun. 34 (2013) 113-116. 
[26] L. Wang, A. Ambrosi, M. Pumera, "Metal-Free" catalytic oxygen reduction reaction on heteroatom-doped graphene is caused by trace metal impurities, Angew. Chem. Int. Ed. 52 (51) (2013) 13818-13821. 